\title{
PRODUCTIVIDAD DE UNA PRADERA NATURAL MEJORADA BAJO LA INFLUENCIA DE SISTEMAS SILVOPASTORALES CON PinUs contorta EN RELACION A UNA SITUACIÓN DE MANEJO GANADERO SIN ÁRBOLES
}

\author{
Sotomayor, Alvaro'; Teuber Osvaldo ${ }^{2}$; Moya Ivan ${ }^{1}$ y Almonacid, Patricio ${ }^{2}$
}

\section{RESUMEN}

Entre los años 2004 y 2008, se realizó un estudio en la Región de Aysén, Chile, para evaluar el desarrollo y composición de una pradera naturalizada, creciendo bajo plantaciones de Pinus contorta Doug. Ex Loud. manejadas bajo ordenación silvopastoral en sistemas homogéneos y en fajas alternadas, en relación a una pradera sin árboles. De acuerdo a los resultados obtenidos en cuatro temporadas de evaluación, se registró una mayor producción de la pradera en el tratamiento silvopastoral en fajas en todas las temporadas evaluadas, con diferencias significativas en relación al tratamiento ganadero.

En relación a la composición de los pastos, también se observa un efecto beneficioso de los árboles sobre las leguminosas y gramíneas. De acuerdo a los resultados obtenidos, existe una mayor presencia de leguminosas en el tratamiento silvopastoral en fajas y tradicional, aunque no existen diferencias significativas con el ganadero. Sí se presentaron diferencias significativas, a favor de los tratamientos silvopastorales, para gramíneas con respecto al ganadero. Esto indica, que la protección de los árboles sobre la pradera benefició el desarrollo de las leguminosas y gramíneas presentes en las temporadas evaluadas. Lo anterior está relacionado con una mayor cobertura de copa, la cual ha beneficiado con su protección el desarrollo de las leguminosas y gramíneas. Al final del periodo de evaluación, la cobertura de copa máxima alcanzada ha sido del $32 \%$ para silvopastoral tradicional y de $31 \%$ para silvopastoral en fajas alternadas.

Además, se evaluó la distribución y composición de los pastos creciendo bajo la influencia de los árboles en tratamientos silvopastorales, comprobándose que las leguminosas prosperaron mejor al abrigo de los árboles, pero que aumentaban su presencia a medida que se alejaban del éstos, entre 2,5 a 5,0 m, con una menor proporción bajo la copa o a un metro de éstas. En relación a las gramíneas, éstas aumentaron su presencia en las cercanías de los árboles, entre 1,0 a $2,5 \mathrm{~m}$.

Por lo anterior, los sistemas silvopastorales se presentan como una buena alternativa para mejorar la productividad pratense, sin descuidar la protección del ambiente.

Palabras clave: Sistemas Agroforestales, Pinus contorta, praderas.

\footnotetext{
${ }_{1}^{1}$ Instituto Forestal (INFOR), asotomay@infor.cl

2 Instituto de Investigaciones Agropecuarias (INIA)
} 


\section{SUMMARY}

Between 2004 and 2008, a study was conducted in the Region of Aysén, Chile, to evaluate the development and composition of a naturalized prairie, growing under Pinus contorta, managed though homogeneous silvopastoral systems and in alternated stripes in relation to a livestock treatment, without trees. According to the results of four years evaluation, there was increased production of prairie in strip silvopastoral treatment in all seasons evaluated, with significant differences related with non trees treatment.

In relation to the composition of the pastures, there is also a beneficial effect of the trees over legume and grasses. According to the results, there is a greater presence of legumes in the traditional and strip silvopastoral treatments, but no significant differences with the non trees treatment. There were significant differences in favor of silvopastoral treatments for grasses in relation with livestock treatment.

Keywords: Agroforestry Systems, Pinus contorta, Grassland 


\section{INTRODUCCIÓN}

La Región de Aysén, Chile, presenta severos contrastes en cuanto a su productividad y sustentabilidad ambiental. Por un lado, algunos predios ganaderos ubicados en suelos planos y profundos, o en lomajes suaves, presentan altas productividades, pero requieren también altas inversiones en fertilización y mejoramiento de praderas y genética animal. Por otro lado, existen miles de hectáreas de suelos de aptitud forestal que han sido descubiertos de bosques naturales para su uso en la agricultura en los últimos dos siglos (Teuber y Ganderatz, 2009).

Gran parte de los suelos descubiertos están siendo utilizados en prácticas ganaderas, de baja productividad, y se encuentran erosionados desde una categoría leve a severa (IRENCORFO, 1979; Teuber y Ganderatz, 2009). El uso de estos suelos mediante prácticas más sustentables es un desafío para esta Región de Chile.

El principal uso de los suelos agrícolas de la Región de Aysén es el de praderas naturales, destinadas al uso ganadero extensivo, con un $88,7 \%$ de la superficie agrícola utilizada, de la cual el $56,5 \%$ pertenece a grandes propietarios (INE, 1997). Esto se contrasta con las menores superficies de plantaciones del país a nivel regional, con solo 43 mil ha (INFOR, 2008).

Las bajas tasas de forestación, se deben a la baja aceptación de los ganaderos respecto de las actividades de forestación tradicional, ya que usualmente la perciben como una competencia con sus usos productivos agrícolas tradicionales.

Una práctica que se ha estado estudiando en los últimos años por INFOR e INIA, instituciones pertenecientes al Ministerio de Agricultura de Chile, es el uso de sistemas agroforestales, principalmente sistemas silvopastorales y cortinas cortavientos, para así reintroducir el árbol en una forma amigable con los sistemas productivos ganaderos y con la cultura productiva de los propietarios de Aysén. De esta forma, se espera reducir el nivel de erosión de los suelos y ayudar a mejorar la productividad de éstos, protegiéndolos del factor erosivo más importante en la región, que es el viento (Teuber y Ganderatz, 2009).

Los sistemas silvopastorales son una forma de aplicación de los sistemas agroforestales y se los define como aquella práctica agroforestal que combina árboles, con forraje y producción de ganado en un mismo sitio, en forma deliberada, con el objetivo de producir alimento para los animales, productos derivados del ganado y productos forestales, como madera y pulpa, y otros como leña, carbón, miel, frutos y hongos (Sotomayor, 1990a).

Algunos de los beneficios que se ha identificado de la aplicación de los sistemas silvopastorales en predios ganaderos $\mathrm{y} / \mathrm{o}$ forestales tradicionales son:

Aprovechar la protección que puede ofrecer el árbol, tanto a los animales como a la pradera, frente a condiciones climáticas adversas (Sotomayor, 1989)

Diversificar la actividad productiva de la mediana y pequeña empresa agrícola, haciendo un uso eficiente y sustentable de los recursos prediales disponibles (Snaydon y Harris, 1979)

Generar flujos de caja anuales y mejorar la liquidez de los agricultores o de la empresa forestal tradicional (Sotomayor, 2009; Murgeitio, 2009)

Reducir el riesgo de incendio y controlar el crecimiento de malezas en plantaciones forestales tradicionales (Murgeitio, 2009)

Mejorar el crecimiento y calidad de la madera producida 
Mejorar la belleza escénica del predio y el valor de la propiedad (Sotomayor y Cabrera, 2008; Sotomayor, 2009)

Protección y mejoramientos de los suelos (Nair, 1987; Murgeitio, 2009).

En un sentido económico, la integración que logra el manejo silvopastoral permite la producción de madera de alta calidad, cuando es aplicado correctamente, generando ingresos de mediano y largo plazo, a la vez de proveer un ingreso de corto plazo, proveniente de las operaciones ganaderas y de raleos intermedios. Estos ingresos permiten, a su vez, financiar los costos asociados al manejo forestal, como son principalmente raleo y poda, y el manejo de los desechos (Sotomayor, 1989; Sotomayor, 1990a).

La incorporación de árboles en sectores destinado al uso exclusivo de pastoreo conforma un sistema sustentable con variados beneficios ambientales, los cuales se relacionan con la protección invernal de los animales, en particular del efecto del viento sobre el descenso de la temperatura; el mejoramiento de la capacidad de retención de humedad del suelo (Mead, 2009); el bombeo de nutrientes desde la parte más profunda del perfil a la porción más superficial; $y$, en general, con el aumento del contenido de materia orgánica del suelo (Sotomayor et al., 2009; Murgeitio, 2009).

Sin embargo, el manejo del sistema silvopastoral es más complejo que un sistema productivo monoespecífico, pues en él se produce la interacción entre los componentes herbáceos, arbóreos (y/o arbustivos) y animal (Garret and Buck, 1997; Garret et al. 2004; Mead, 2009; Sotomayor et al., 2009). En este sentido, los manejos asociados deben estar orientados a minimizar la competencia entre los estratos arbóreos y herbáceos, así como el daño potencial que puede provocar la presencia de animales en las plantaciones forestales (Lewis, 1983).

En términos generales, el mayor conflicto entre los estratos arbóreo y la herbáceo se relaciona con la competencia por agua y nutrientes, especialmente en el establecimiento de las plantaciones forestales, y con la competencia por luz directa (Anderson et al., 1988), la que aumenta proporcionalmente con el desarrollo de los árboles y el cierre del dosel (Mead, 2009).

Por otro lado, el efecto de los animales se relaciona directamente con el ramoneo y pisoteo de las plantas, situación que es especialmente sensible durante los primeros años de vida de la planta (Monfore, 1983; Sotomayor, 1990b).

Si bien es reconocido en diversos estudios que el desarrollo y cierre del dosel arbóreo, estrechamente ligado al desarrollo de las especies y el manejo forestal, provoca una disminución de la producción de forraje, se ha observado que bajo determinadas condiciones este efecto es compensado por la acción protectora que ejercen los árboles sobre los pastos (Solangarachchi y Harper, 1997; Benavides et al., 2009).

La acción protectora de los árboles disminuye la velocidad del viento y atenúa la acción del frío en el invierno y de las altas temperaturas de verano y reduce la pérdida de humedad durante el verano, situación que permite que la cubierta herbácea permanezca por más tiempo disponible para el ganado (Solangarachchi y Harper, 1997; Sotomayor et al., 2009)

\section{OBJETIVO}

Comparar la productividad de una pradera natural mejorada, bajo la influencia de manejo silvopastoral con la especie Pinus contorta, en relación a una situación de manejo ganadero sin árboles. 


\section{MATERIAL Y METODO}

\section{Diseño y Tratamientos}

Para evaluar la factibilidad de uso de sistemas silvopastorales con coníferas de crecimiento lento (Pinus ponderosa, Pinus contorta y Pseudotzuga menziesii) en la Región de Aysén, este estudio consideró la implementación de módulos silvopastorales y ganaderos, con el objeto de evaluar la productividad de estos y su validación bajo las condiciones edafoclimáticas regionales.

Los tratamientos instalados, su diseño y superficie, para dar respuestas a las interrogantes planteadas en objetivo, relacionadas con la producción pratense bajo tres esquemas de manejo, son descritos en el Cuadro $N^{\circ} 1$. Los ensayos fueron instalados durante el año 2004, y para efectos de evaluación de la pradera, se dispuso parcelas de evaluación por tratamiento con un diseño al azar, de acuerdo a lo expuesto en el cuadro mencionado.

\section{Cuadro $\mathrm{N}^{\circ} 1$ \\ SUPERFICIE Y PARCELAS DE EVALUACIÓN PRATENSE POR TRATAMIENTO}

\begin{tabular}{|l|c|c|c|c|}
\hline \multirow{2}{*}{ Tratamientos } & \multicolumn{2}{|c|}{ Superficie } & \multicolumn{2}{c|}{ Parcelas Evaluación } \\
\cline { 2 - 5 } & $\begin{array}{c}\text { Total } \\
\text { (ha) }\end{array}$ & $\begin{array}{c}\text { Efectiva* } \\
\text { (ha) }\end{array}$ & (No) ${ }^{* *}$ & $\mathbf{( m}^{\mathbf{2}} \mathbf{~}$ \\
\hline $\begin{array}{l}\text { T 2 Silvopastoral tradicional, con pradera } \\
\text { natural mejorada mediante fertilización. }\end{array}$ & 5,3 & 3,94 & $7(\mathrm{~J})$ & 0,50 \\
\hline $\begin{array}{l}\text { T 3 Silvopastoral en fajas alternadas, con } \\
\text { pradera natural mejorada mediante fertilización. }\end{array}$ & 5,5 & 4,19 & $7(\mathrm{~J})$ & 0,50 \\
\hline $\begin{array}{l}\text { T 4 Ganadero tradicional, con pradera natural } \\
\text { mejorada mediante fertilización, sin árboles. }\end{array}$ & 4,3 & 4,30 & $7(\mathrm{~J})$ & 0,50 \\
\hline
\end{tabular}

*: Superficie efectiva de pradera, descontando área cubierta con desechos de poda y raleo.

**: Tipo de parcela, J: Jaula de exclusión de 50 ×50 x $100 \mathrm{~cm}$.

La información para la evaluación de la pradera fue tomada en cuatro temporadas: 2004 2005, 2005-2006, 2006-2007 y 2007-2008. La temporada se calcula entre octubre a abril-mayo de cada año, es decir primavera a otoño.

\section{Parámetros Evaluados}

\section{-Materia Seca}

La materia seca, consiste en la materia generada por el secado a $60^{\circ} \mathrm{C}$ en hornos del material pratense verde cortado en las jaulas de evaluación. Las evaluaciones para determinar la curva de crecimiento de la pradera en materia seca, consisten en la toma de muestras seriadas, cada 30 días, entre diciembre y mayo de cada temporada, utilizando la metodología de jaulas de exclusión.

Cada una de las muestras es pesada para determinar su peso verde y posteriormente secada para establecer la cantidad de materia seca ( $\mathrm{kg} \mathrm{MS} \mathrm{/} \mathrm{ha).}$ 
Se utilizó un diseño experimental de jaulas de exclusión dispuestas al azar dentro de los tratamientos T2, T3 y T4, las cuales variaron de ubicación en cada temporada. Para las evaluaciones, se utilizaron 7 jaulas de exclusión de $50^{*} 50^{*} 100 \mathrm{~cm}$ por tratamiento, y 2 segadoras eléctricas para el corte de muestras.

\section{-Composición de la Pradera}

Se evaluó la distribución de la composición botánica de la pradera en relación a componente forestal mediante análisis de composición florística para determinar la participación relativa de las principales especies de gramíneas, leguminosas, de malezas y la presencia de material muerto. Estos análisis se fueron realizados en el Laboratorio de Recursos Naturales del CRI Tamel Aike de INIA, Comuna de Coyhaique.

Para la determinación de la composición de la pradera y su relación con el componente forestal para los casos silvopastorales, se utilizó un sistema de transectos de $5 \mathrm{~m}$ de ancho por 22 $\mathrm{m}$ de largo, para estudiar el comportamiento de la pradera bajo influencia arbórea en los dos tratamientos silvopastorales, y como testigo en el tratamiento ganadero. Se estableció 5 transectos por cada tratamiento, y 6 parcelas de medición de 50*50*100 cm por transecto. Estos transectos fueron cercados para excluirlos del uso animal.

Para la medición de la pradera en el tratamiento silvopastoral tradicional, se establecieron parcelas de medición, similar a lo expuesto anteriormente para materia seca, ubicadas bajo diferentes condiciones: condición de bajo copa, semi-sombra y sector sin influencia directa de la copa, según la siguiente descripción (Figura $\mathrm{N}^{\circ} 1$ ):

Parcela T1bce:

Parcela T1bco:

Parcela T1ss1:

Parcela T1ss2:

Parcela T1a1:

Parcela T1a2:
Bajo copa, ubicación este: a 0,3 m del fuste del árbol.

Bajo copa, ubicación oeste: a 0,3 m del fuste del árbol.

Semi-sombra, ubicación este: a 1,5 m de la proyección de copa del árbol.

Semi-sombra, ubicación oeste: a 1,5 m de la proyección de copa del árbol.

Sin cobertura, ubicación este: a 2,5 m de la proyección de copa del árbol.

Sin cobertura, ubicación oeste: a 2,5 m de la proyección de copa del árbol.

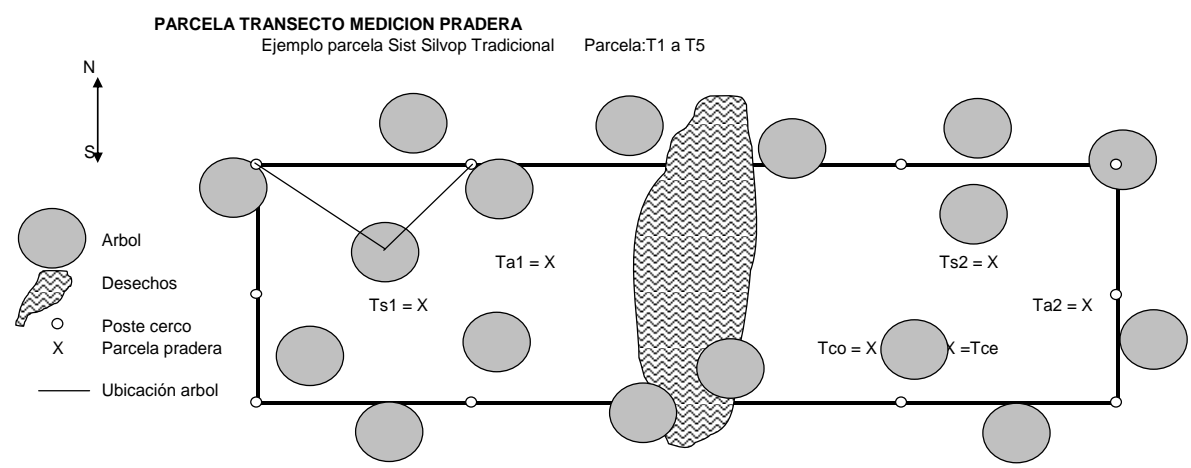

Figura $\mathrm{N}^{\circ} 1$

DESCRIPCIÓN PARCELA DE EVALUACIÓN DISTRIBUCIÓN DE LA COMPOSICIÓN BOTÁNICA DE PRADERA NATURAL, EN TRATAMIENTO SILVOPASTORAL TRADICIONAL 
Para tratamiento en fajas, el transecto considera el efecto a cada lado de las fajas de árboles (orientación este y oeste), y se midió la producción de la pradera, según la siguiente descripción (Figura $\mathrm{N}^{\circ} 2$ ):
Parcela Fe1:
Parcela Fe4:
Parcela Fe9:
Parcela Fo1:
Parcela Fo4:
Parcela Fo9:

\begin{abstract}
Ubicación este, a $1 \mathrm{~m}$ de la de proyección de copa Ubicación este, a $4 \mathrm{~m}$ de la de proyección de copa Ubicación este, a $9 \mathrm{~m}$ de la de proyección de copa Ubicación oeste, a $1 \mathrm{~m}$ de la de proyección de copa Ubicación oeste, a 4 m de la de proyección de copa Ubicación oeste, a $9 \mathrm{~m}$ de la de proyección de copa
\end{abstract}

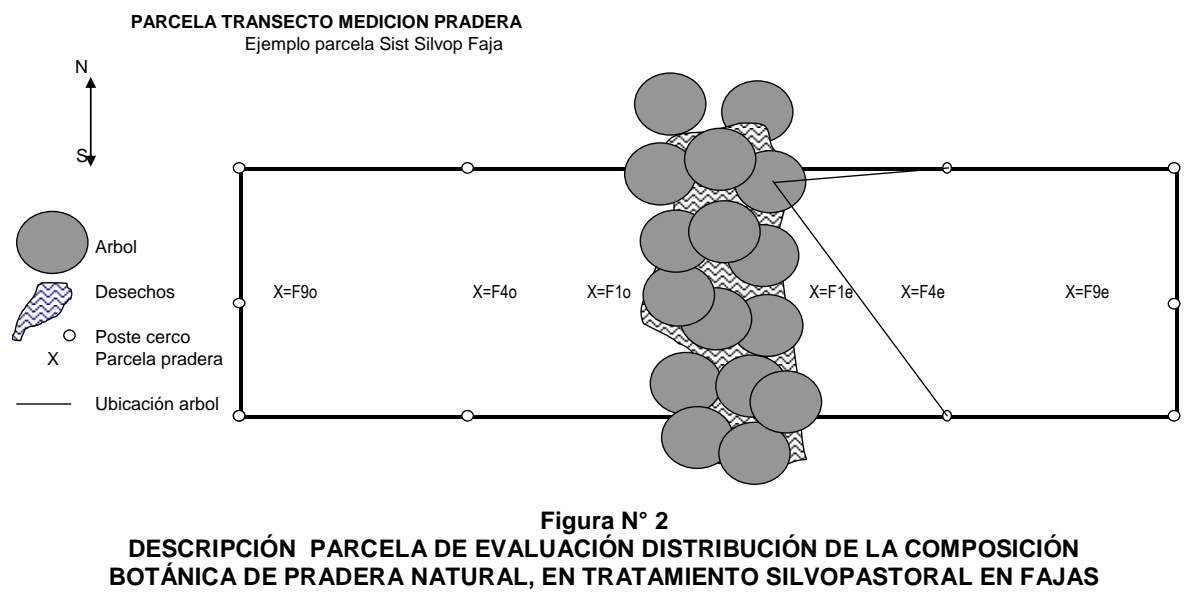

Para realizar los análisis estadísticos, se utilizó un análisis de varianza tradicional, dado que se cumplen los criterios básicos para este; los datos presentan una distribución normal, son independientes y hay heterogeneidad de varianza.

Para el análisis de la variable producción pratense $\left(\mathrm{kg} \mathrm{MS} \mathrm{ha}^{-1}\right)$ y composición de la pradera, se realizó un análisis de mediciones anuales, con jaulas de exclusión ubicadas en lugares diferentes y al azar cada año.

Estas mediciones son independientes unas de otras, lo que origina cumplimiento de la independencia de datos (las mediciones del año 2 no dependen de las mediciones del año 1 y así sucesivamente), dado lo cual se usó el análisis de varianza tradicional.

Este análisis permite identificar tanto el efecto medio del tratamiento como el efecto del tiempo y la interacción tiempo por tratamiento, que corresponden a las tres hipótesis evaluadas, y que por lo tanto generan tres valores $\mathrm{p}$. El modelo de análisis corresponde al modelo expuesto en fórmula 1.

$$
\mathbf{Y}=\mu+T+t+\left(T^{*} t\right)+P+E \quad \text { Formula 1. Modelo de análisis estadístico }
$$

Donde: $\quad \mathrm{Y}=$ Modelo

$\mu=$ Constante 


$$
\begin{aligned}
& \mathrm{T}=\text { Tratamiento } \\
& \mathrm{t}=\text { Tiempo } \\
& \mathrm{P}=\text { Parcela } \\
& \mathrm{E}=\text { Error }
\end{aligned}
$$

\section{RESULTADOS}

\section{Productividad Pradera en Materia Seca}

$\mathrm{N}^{\circ} 2$ y Figura $\mathrm{N}^{\circ} 3$.

Los resultados de producción de la pradera $\left(\mathrm{kg} \mathrm{MS} \mathrm{ha}^{-1}\right)$ son presentados en el Cuadro

Cuadro $\mathrm{N}^{\circ} 2$

PRODUCCIÓN PRADERA TEMPORADA 2004-2005 A 2007-2008

\begin{tabular}{|l|r|r|r|r|}
\hline \multirow{2}{*}{ Tratamiento } & \multicolumn{4}{|c|}{$\begin{array}{r}\text { Producción Pradera por Temporada } \\
\text { (kg MS ha }\end{array}$} \\
\cline { 2 - 5 } & $\mathbf{2 0 0 4 - 2 0 0 5}$ & $\mathbf{2 0 0 5 - 2 0 0 6}$ & $\mathbf{2 0 0 6 - 2 0 0 7}$ & \multicolumn{1}{|c|}{$\mathbf{2 0 0 7 - 2 0 0 8}$} \\
\hline T2 Silvolpastoral Tradicional & 1485,7 & 6109,7 & 4153,2 & 4330,9 \\
\hline T3 Silvopastoral en Fajas & 2684,9 & 7181,6 & 6394,5 & 5359,7 \\
\hline T4 Ganadero Tradicional & 2452,1 & 3832,0 & 3874,1 & 3513,6 \\
\hline
\end{tabular}

En las cuatro temporadas de evaluación, la producción de la pradera en materia seca por hectárea en el sistema silvopastoral en fajas ha sido la que alcanzó las mayores producciones.

La producción de la pradera en el sistema silvopastoral tradicional ha evolucionado positivamente a partir de la segunda temporada, por sobre el sistema ganadero. Se ha observado un rápido incremento en la producción pratense en este tratamiento silvopastoral, a partir de la segunda temporada de evaluación, debido a un mayor tiempo para expresar el potencial de desarrollo de la pradera después de la apertura del dosel superior.

Finalmente, el sistema ganadero tradicional ha sido el de menor desarrollo de la pradera.

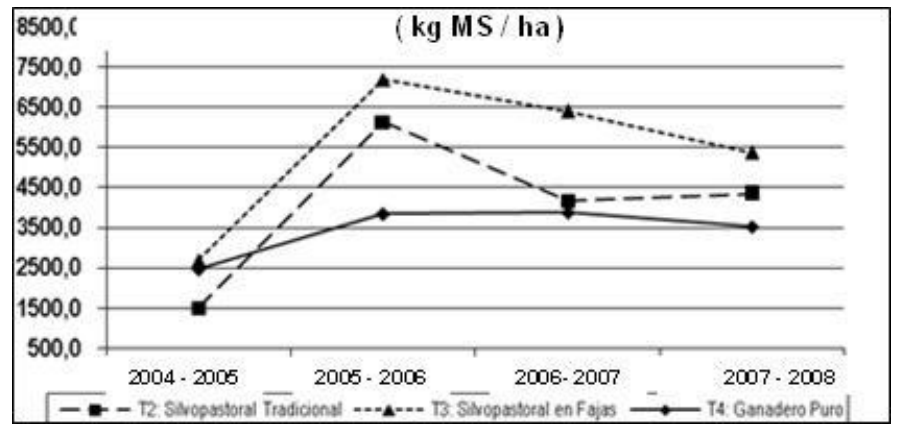

Figura $\mathrm{N}^{\circ} 3$

EVOLUCIÓN PRODUCCIÓN PRADERA, AÑOS 2004 A 2008 
En los resultados expuestos en el Cuadro $\mathrm{N}^{\circ} 3$ se observa que el efecto tratamiento, tiempo (o temporada), y tratamiento*tiempo son significativos para la variable MS.

Usando el test de Scott \& Knott, a un $\propto<=0,05$, para el efecto tratamiento*tiempo, se obtuvieron los siguientes resultados (Cuadro $\left.\mathrm{N}^{\circ} 4\right)$.

Para el efecto tratamiento*tiempo se observa en los resultados expuestos que en la primera temporada de producción no hubo diferencias significativas.

A partir de la segunda temporada, los tratamientos silvopastorales, especialmente el tratamiento en fajas, comenzaron a mostrar una buena productividad, con diferencias significativas en relación al ganadero. Para las dos últimas temporadas de evaluación, el tratamiento en fajas mostró una mayor producción de la pradera, con diferencias significativas en relación a los otros dos tratamientos.

Cuadro $\mathrm{N}^{\circ} 3$

RESULTADO DE ANÁLISIS DE VARIANZA PARA MS

\begin{tabular}{|c|c|c|c|c|c|}
\hline F.V. & SC & gl & CM & $\mathbf{F}$ & p-valor \\
\hline Tratamiento & 57303617 & 2 & 28651808 & 14,99 & $<0,0001$ \\
\hline Tiempo & 139135024 & 3 & 46378341 & 24,26 & $<0,0001$ \\
\hline Tratamiento*Tiempo & 27180681 & 6 & 4530114 & 2,37 & 0,0383 \\
\hline Error & 135746021 & 71 & 1911916 & & \\
\hline Total & 359631714 & 82 & & & \\
\hline
\end{tabular}

Cuadro $\mathrm{N}^{\circ} 4$

RESULTADO DE ANÁLISIS DE VARIANZA PARA EFECTO TRATAMIENTO^TIEMPO EN MS

\begin{tabular}{|l|c|r|c|c|}
\hline TRATAMIENTO & Temporada & Medias & $\mathbf{n}$ & $\begin{array}{c}\text { Significancia } \\
\mathbf{p}<=\mathbf{0 , 0 5 ^ { * }}\end{array}$ \\
\hline T3. Sil. Fajas & $2004-2005$ & 2684,87 & 7 & $\mathrm{C}$ \\
\hline T4. Ganadero & $2004-2005$ & 2452,11 & 7 & $\mathrm{C}$ \\
\hline T2. Sil. Tradicional & $2004-2005$ & 1485,73 & 7 & $\mathrm{C}$ \\
\hline T3. Sil. Fajas & $2005-2006$ & 7181,57 & 7 & $\mathrm{~A}$ \\
\hline T2. Sil. Tradicional & $2005-2006$ & 6109,69 & 7 & $\mathrm{~A}$ \\
\hline T4. Ganadero & $2005-2006$ & 3832,06 & 7 & $\mathrm{~B}$ \\
\hline T3. Sil. Fajas & $2006-2007$ & 6394,53 & 7 & $\mathrm{~A}$ \\
\hline T2. Sil. Tradicional & $2006-2007$ & 4153,24 & 7 & $\mathrm{~B}$ \\
\hline T4. Ganadero & $2006-2007$ & 3874,07 & 7 & $\mathrm{~B}$ \\
\hline T3. Sil. Fajas & $2007-2008$ & 5359,67 & 7 & $\mathrm{~A}$ \\
\hline T2. Sil. Tradicional & $2007-2008$ & 4330,94 & 7 & $\mathrm{~B}$ \\
\hline T4. Ganadero & $2007-2008$ & 3513,58 & 6 & $\mathrm{~B}$ \\
\hline
\end{tabular}

*Letras distintas indican diferencias significativas $(p<=0,05)$

En general, la mejor producción en todas las temporadas fue para el tratamiento en fajas. La peor temporada de producción fue el primer periodo de evaluación, 2004-2005, para los tres tratamientos, debido a que fue el primer año de producción después de la fertilización, y después de la apertura del dosel para los tratamientos silvopastorales. La mejor fue la segunda temporada 
de evaluación, producto de las mismas razones expuestas, fertilización y reducción de cobertura de copa.

\section{Composición de la Pradera}

Para analizar la composición de la pradera y su evolución en los años de estudio, se presenta los datos por cada tipo de pasto presente en la pradera y por temporada.

\section{- Leguminosas}

En el Cuadro $N^{\circ} 5$ se entrega los resultados de la evolución de las leguminosas por tratamiento y temporada, con presencia mayoritaria de trébol blanco (Trifolium repens), por tratamiento y año.

Cuadro $\mathrm{N}^{\circ} 5$

\section{COMPOSICIÓN DE LEGUMINOSAS POR TRATAMIENTO Y TEMPORADA}

\begin{tabular}{|l|l|r|r|r|r|}
\hline \multirow{2}{*}{ Tratamiento } & \multirow{2}{*}{ Tipo Pasto } & \multicolumn{4}{|c|}{ Composición de Leguminosas } \\
\cline { 3 - 6 } & & 2004-2005 & $\mathbf{2 0 0 5 - 2 0 0 6}$ & $\mathbf{2 0 0 6 - 2 0 0 7}$ & 2007-2008 \\
\hline $\begin{array}{l}\text { T 2 Silvopastoral } \\
\text { Tradicional }\end{array}$ & Leguminosas & 11,3 & 33,3 & 32,8 & 38,8 \\
\hline T 3 Silvopastoral en Fajas & Leguminosas & 22,1 & 41,4 & 38,4 & 36,0 \\
\hline T 4 Ganadero Tradicional & Leguminosas & 10,1 & 37,8 & 41,4 & 26,3 \\
\hline
\end{tabular}

La evolución de leguminosas en los tratamientos (Cuadro $N^{\circ} 5$ y Figura $N^{\circ} 4$ ) ha sido positiva producto de la fertilización aplicada en el primer año de ensayo con azufre y nitrógeno, de un correcto manejo animal y de la apertura del bosque. En la temporada siguiente a la fertilización los tres tratamientos tuvieron un similar incremento porcentual de leguminosas. En la temporada 2007-2008 se observa que el tratamiento silvopastoral tradicional siguió incrementando la presencia de leguminosas y los otros dos tratamientos disminuyeron a 36 y $26 \%$ para silvopastoral en fajas y ganadero, respectivamente.

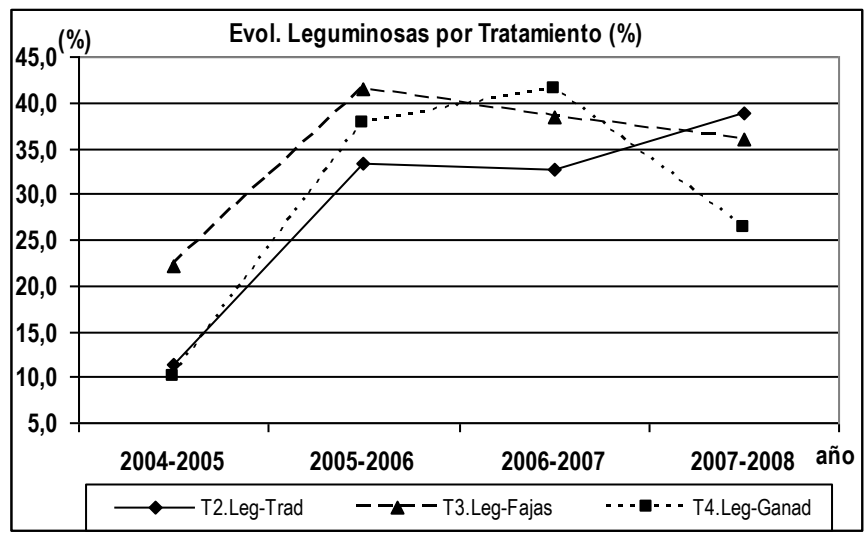

Figura $N^{\circ} 4$

EVOLUCIÓN COMPOSICIÓN DE LEGUMINOSAS POR TRATAMIENTO Y AÑO 
Se emplearon los mismos análisis estadísticos utilizados para la variable materia seca y los resultados son los indicados en el Cuadro $N^{\circ} 6$, donde se observa que el efecto modelo y temporada son significativos, pero el efecto tratamiento y tratamiento*temporada no son significativos para la variable composición de leguminosas.

De acuerdo a lo anterior y a los resultados que contiene el Cuadro $\mathrm{N}^{\circ} 7$, no existen diferencias significativas en el tiempo para los tratamientos en cuanto a la composición de leguminosas, aunque los tratamientos silvopastorales presentan proporciones levemente superiores de este tipo de pasto, especialmente trébol blanco.

Cuadro $N^{\circ} 6$

RESULTADO DE ANÁLISIS DE VARIANZA PARA COMPOSICIÓN DE LEGUMINOSAS

\begin{tabular}{|c|c|c|c|c|c|}
\hline Efecto & SC & gl & CM & $\mathbf{F}$ & p-valor \\
\hline Tratamiento & 545,07 & 2 & 272,53 & 1,38 & 0,2583 \\
\hline Temporada & 6773,44 & 3 & 2257,81 & 11,44 & $<0,0001$ \\
\hline Tratamiento*Temporada & 1089,98 & 6 & 181,66 & 0,92 & 0,4857 \\
\hline Error & 13217,50 & 67 & 197,28 & & \\
\hline Total & 21611,60 & 78 & & & \\
\hline
\end{tabular}

Cuadro $\mathrm{N}^{\circ} 7$

RESULTADO DE ANÁLISIS DE VARIANZA PARA EFECTO TRATAMIENTO EN LEGUMINOSAS

\begin{tabular}{|l|r|r|c|}
\hline Tratamiento & Medias & $\mathbf{n}$ & $\begin{array}{c}\text { Significancia } \\
\mathbf{p}<=\mathbf{0 , 0 5 ^ { * }}\end{array}$ \\
\hline T3 Silvopastoral en Fajas & 34,49 & 27 & $\mathrm{~A}$ \\
\hline T2 Silvopastoral Tradicional & 29,01 & 27 & $\mathrm{~A}$ \\
\hline T4 Ganadero Tradicional & 28,87 & 25 & $\mathrm{~A}$ \\
\hline
\end{tabular}

*Letras distintas indican diferencias significativas $(p<=0,05)$

La única temporada donde se presentaron diferencias entre tratamientos fue la inicial, no presentándose diferencias en la últimas tres temporadas de evaluación (Cuadro № 8). Esto último, producto del efecto de la fertilización y un buen manejo de pradera y pastoral.

\section{Cuadro $\mathrm{N}^{\circ} 8$}

RESULTADO DE ANÁLISIS DE VARIANZA PARA EFECTO TEMPORADA EN LEGUMINOSAS

\begin{tabular}{|c|r|c|c|}
\hline Temporada & \multicolumn{1}{|c|}{ Medias } & $\mathbf{n}$ & $\begin{array}{c}\text { Significancia } \\
\mathbf{p}<=\mathbf{0 , 0 5}\end{array}$ \\
\hline $2006-2007$ & 37,53 & 21 & $\mathrm{~A}$ \\
\hline $2005-2006$ & 37,48 & 20 & $\mathrm{~A}$ \\
\hline $2007-2008$ & 33,68 & 20 & $\mathrm{~A}$ \\
\hline $2004-2005$ & 14,49 & 18 & $\mathrm{~B}$ \\
\hline
\end{tabular}

*Letras distintas indican diferencias significativas $(p<=0,05)$ 


\section{-Gramíneas}

La principal gramínea presente en los tratamientos es pasto ovillo (Dactylis glomerata), la cual presentó un comportamiento similar en los tratamientos silvopastorales en las cuatro primeras temporadas (Cuadro $\mathrm{N}^{\circ} 9$ y Figura $\mathrm{N}^{\circ} 5$ ).

En la temporada 2005-2006, en el tratamiento ganadero se observa una fuerte disminución de gramíneas, que es coincidente con el aumento de las leguminosas en dicho tratamiento y temporada, para después recuperarse en las temporadas siguientes (Cuadro $\mathrm{N}^{\circ} 9$ ).

En los tratamientos silvopastorales, en la segunda temporada de crecimiento se produjo un aumento en la composición de gramíneas, al igual que en leguminosas para esa temporada. En la temporada 2006-2007 las gramíneas aumentaron en los tres tratamientos, y en la última temporada disminuyeron en T2 y T3 producto de una buena colonización de leguminosas, y aumentó en T4 producto de la disminución de las leguminosas en dicho tratamiento (Figura $\mathrm{N}^{\circ} 5$ ).

\section{Cuadro $\mathrm{N}^{\circ} 9$ \\ COMPOSICIÓN DE GRAMÍNEAS POR TRATAMIENTO Y AÑO}

\begin{tabular}{|c|c|c|c|c|c|}
\hline \multirow[t]{2}{*}{ Tratamiento } & \multirow{2}{*}{ Tipo Pasto } & \multicolumn{4}{|c|}{$\begin{array}{c}\text { Composición de Gramíneas } \\
(\%)\end{array}$} \\
\hline & & 2004-2005 & $2005-2006$ & $2006-2007$ & 2007-2008 \\
\hline $\begin{array}{l}\text { T } 2 \text { Silvopastoral } \\
\text { Tradicional }\end{array}$ & Gramíneas & 16,1 & 31,8 & 37,2 & 25,4 \\
\hline $\begin{array}{l}\text { T } 3 \text { Silvopastoral } \\
\text { en Fajas }\end{array}$ & Gramíneas & 18,9 & 31,2 & 37,8 & 33,8 \\
\hline $\begin{array}{l}\text { T } 4 \text { Ganadero } \\
\text { Tradicional }\end{array}$ & Gramíneas & 30,2 & 15,5 & 22,1 & 28,3 \\
\hline
\end{tabular}

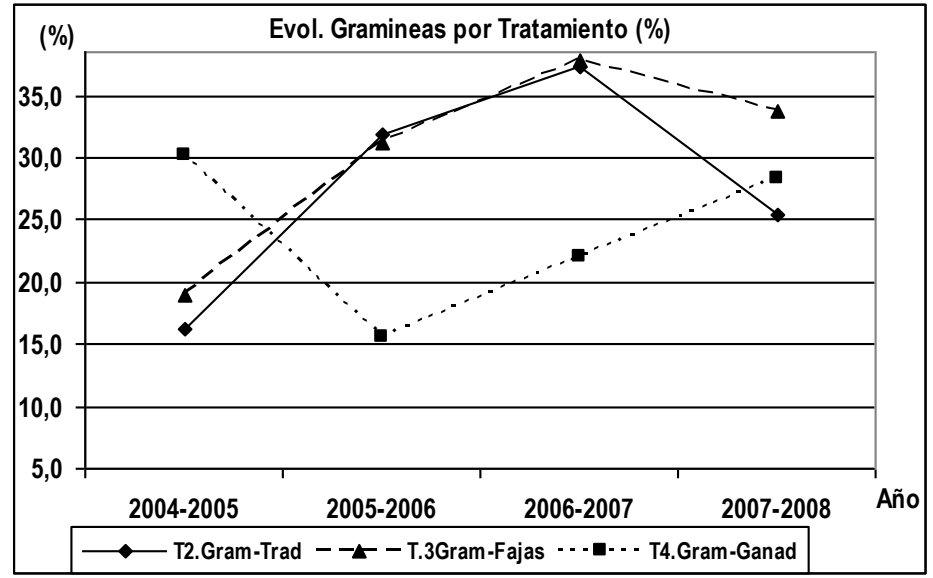

Figura $\mathbf{N}^{\circ} 5$

EVOLUCIÓN COMPOSICIÓN DE GRAMÍNEAS POR TRATAMIENTO Y AÑO 
Como se observa en los resultados expuestos en el Cuadro $N^{\circ} 10$, el efecto tratamiento fue significativo, pero el efecto tiempo y tratamiento*tiempo no son significativos para la variable composición de gramíneas.

Los resultados que aparecen en el Cuadro $N^{\circ} 11$ y la Figura $N^{\circ} 5$ indican que no existen diferencias significativas entre los tratamientos silvopastorales en el tiempo para gramíneas, pero si entre estos y el tratamiento ganadero.

Cuadro $\mathrm{N}^{\circ} 10$

RESULTADO ANÁLISIS DE VARIANZA PARA COMPOSICIÓN DE GRAMÍNEAS

\begin{tabular}{|c|c|c|c|c|c|}
\hline Efectos & Sc & gl & CM & $\mathbf{F}$ & p-valor \\
\hline Tratamiento & 1843,05 & 2 & 921,52 & 7,48 & 0,0012 \\
\hline Tiempo & 671,97 & 3 & 223,99 & 1,82 & 0,1523 \\
\hline Tratamiento*Tiempo & 804,35 & 6 & 134,06 & 1,09 & 0,3787 \\
\hline Error & 8256,43 & 67 & 123,23 & & \\
\hline Total & 11605,52 & 78 & & & \\
\hline
\end{tabular}

Cuadro $\mathrm{N}^{\circ} 11$

RESULTADO ANÁLISIS DE VARIANZA PARA EFECTO TRATAMIENTO DE GRAMÍNEAS

\begin{tabular}{|l|r|c|c|}
\hline Tratamiento & Medias & $\mathbf{n}$ & $\begin{array}{c}\text { Significancia } \\
\mathbf{p}<=\mathbf{0 , 0 5 ^ { * }}\end{array}$ \\
\hline T 3 Silvopastoral en Fajas & 32,54 & 27 & $\mathrm{~A}$ \\
\hline T 2 Silvopastoral Tradicional & 30,56 & 27 & $\mathrm{~A}$ \\
\hline T 4 Ganadero Tradicional & 21,29 & 25 & $\mathrm{~B}$ \\
\hline
\end{tabular}

*Letras distintas indican diferencias significativas $(p<=0,05)$

En el Cuadro $\mathrm{N}^{\circ} 12$ se observa que el efecto tiempo resultó no significativo, con una producción similar en las temporadas evaluadas.

\section{Cuadro $N^{\circ} 12$}

RESULTADO ANÁLISIS DE VARIANZA PARA EFECTO TIEMPO EN GRAMÍNEAS

\begin{tabular}{|c|r|c|c|}
\hline Temporada & Medias & $\mathbf{n}$ & Significancia $\mathbf{p}<=\mathbf{0 , 0 5}$ \\
\hline $2006-2007$ & 32,35 & 21 & $\mathrm{~A}$ \\
\hline $2007-2008$ & 29,19 & 20 & $\mathrm{~A}$ \\
\hline $2005-2006$ & 26,17 & 20 & $\mathrm{~A}$ \\
\hline $2004-2005$ & 24,79 & 18 & $\mathrm{~A}$ \\
\hline
\end{tabular}

*Letras distintas indican diferencias significativas $(p<=0,05)$

\section{-Malezas}

En relación a las malezas, en los tratamientos silvopastorales no aumentó su presencia en las temporadas evaluadas (Cuadro $\mathrm{N}^{\circ} 13$ ). La razón aparente es que luego de la apertura del bosque inicial, tanto las gramíneas como leguminosas colonizaron rápidamente el terreno, no permitiendo el emplazamiento de malezas en una mayor proporción en estos tratamientos. 
Cuadro $\mathrm{N}^{\circ} 13$

PRESENCIA DE MALEZAS POR TRATAMIENTO Y AÑO

\begin{tabular}{|c|c|c|c|c|c|}
\hline \multirow{2}{*}{ Tratamiento } & \multirow{2}{*}{ Tipo Pasto } & \multicolumn{4}{|c|}{$\begin{array}{c}\text { Composición de malezas } \\
(\%)\end{array}$} \\
\hline & & 2004-2005 & $2005-2006$ & 2006-2007 & $2007-2008$ \\
\hline T 2 Silvopastoral Tradicional & Malezas & 16,1 & 20,7 & 21,9 & 18,4 \\
\hline T 3 Silvopastoral en Fajas & Malezas & 18,9 & 17,1 & 14,2 & 17,1 \\
\hline T 4 Ganadero Tradicional & Malezas & 30,2 & 25,7 & 30,8 & 31,4 \\
\hline
\end{tabular}

En el tratamiento ganadero, las malezas se mantuvieron con una proporción constante desde el inicio del estudio, sin mostrar variaciones significativas en el tiempo (Figura $N^{\circ} 6$ ), aunque con valores superiores a los tratamientos silvopastorales.

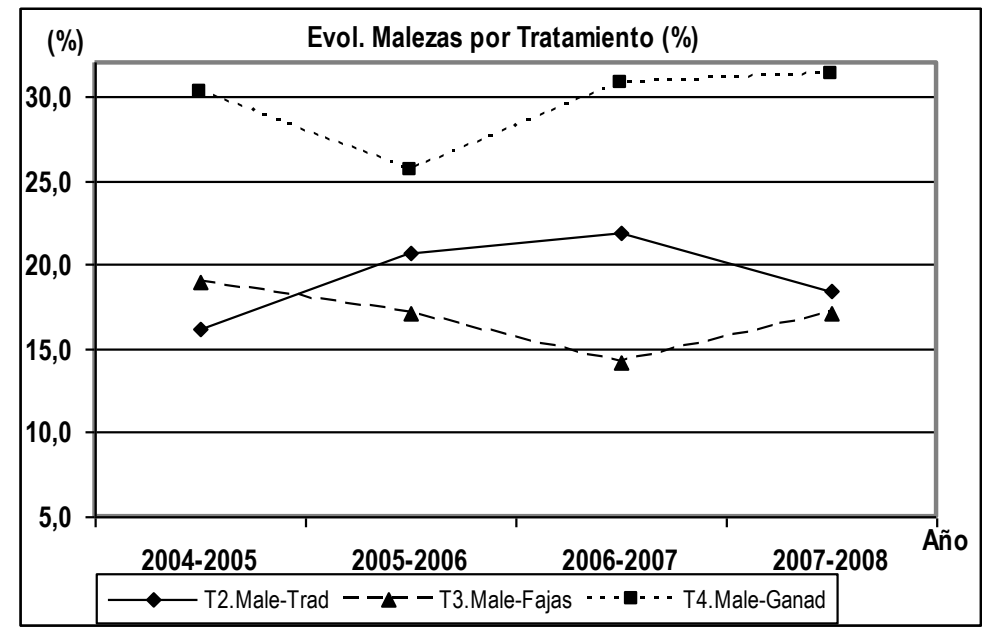

Figura $\mathrm{N}^{\circ} 6$

EVOLUCIÓN COMPOSICIÓN DE MALEZAS POR TRATAMIENTO Y AÑO

En los resultados expuestos en el Cuadro $\mathrm{N}^{\circ} 14$ se observa que el efecto tratamiento es significativo, pero los efectos temporada y tratamiento*temporada no son significativos para la variable composición de malezas.

Cuadro $\mathrm{N}^{\circ} 14$

RESULTADO ANÁLISIS DE VARIANZA PARA COMPOSICIÓN DE MALEZAS

\begin{tabular}{|c|c|c|c|c|c|}
\hline F.V. & SC & gl & CM & $\mathbf{F}$ & p-valor \\
\hline Tratamiento & 2299,72 & 2 & 1149,86 & 12,38 & $<0,0001$ \\
\hline Temporada & 17,07 & 3 & 5,69 & 0,06 & 0,9800 \\
\hline Tratamiento*Temporada & 312,23 & 6 & 52,04 & 0,56 & 0,7604 \\
\hline Error & 6224,48 & 67 & 92,90 & & \\
\hline Total & 8884,10 & 78 & & & \\
\hline
\end{tabular}


Según datos expuestos en el Cuadro $\mathrm{N}^{\circ} 15$ y la Figura $\mathrm{N}^{\circ} 6$, no existen diferencias significativas para el efecto tratamiento para malezas entre los tratamientos silvopastorales, pero si entre estos y el tratamiento ganadero, donde existe mayor presencia de malezas.

Cuadro $\mathrm{N}^{\circ} 15$

RESULTADO ANÁLISIS DE VARIANZA PARA EFECTO TRATAMIENTO EN MALEZAS

\begin{tabular}{|l|c|c|c|}
\hline Tratamiento & Medias & $\mathbf{n}$ & $\begin{array}{c}\text { Significancia } \\
\mathbf{p}<=\mathbf{0 , 0 \mathbf { 0 5 } ^ { * }}\end{array}$ \\
\hline T 4 Ganadero Tradicional & 29,50 & 25 & $\mathrm{~A}$ \\
\hline T 2 Silvopastoral Tradicional & 19,29 & 27 & $\mathrm{~B}$ \\
\hline T 3 Silvopastoral en Fajas & 16,86 & 27 & $\mathrm{~B}$ \\
\hline
\end{tabular}

*Letras distintas indican diferencias significativas $(p<=0,05)$

Para el efecto temporada, no hubo diferencias significativas (Cuadro $\mathrm{N}^{\circ} 16$ ).

Cuadro $\mathrm{N}^{\circ} 16$

RESULTADO ANÁLISIS DE VARIANZA PARA EFECTO TIEMPO EN MALEZAS

\begin{tabular}{|c|r|c|c|}
\hline \multicolumn{1}{|l|}{ Temporada } & \multicolumn{1}{c|}{ Medias } & $\mathbf{n}$ & Significancia $\mathbf{p}<\mathbf{= 0 , \mathbf { 0 5 } ^ { \star }}$ \\
\hline $2006-2007$ & 22,30 & 21 & $\mathrm{~A}$ \\
\hline $2007-2008$ & 22,29 & 20 & $\mathrm{~A}$ \\
\hline $2004-2005$ & 21,77 & 18 & $\mathrm{~A}$ \\
\hline $2005-2006$ & 21,17 & 20 & $\mathrm{~A}$ \\
\hline
\end{tabular}

*Letras distintas indican diferencias significativas $(p<=0,05)$

\section{-Material Muerto}

El contenido de material muerto en los sistemas silvopastorales, al inicio del ensayo estaba constituido por acículas de pino, ramillas, ramas gruesas y conos. En el caso del sector ganadero era material muerto de la pradera y otros vegetales leñosos.

Cuadro $\mathrm{N}^{\circ} 17$

PRESENCIA DE MATERIAL MUERTO POR TRATAMIENTO Y AÑO

\begin{tabular}{|l|c|r|r|r|r|}
\hline \multirow{2}{*}{ Tratamiento } & \multirow{2}{*}{ Tipo Pasto } & \multicolumn{3}{|c|}{ Composición Pradera } \\
\cline { 3 - 6 } & & $\mathbf{2 0 0 4 - 2 0 0 5}$ & $\mathbf{2 0 0 5 - 2 0 0 6}$ & $\mathbf{2 0 0 6 - 2 0 0 7}$ & $\mathbf{2 0 0 7 - 2 0 0 8}$ \\
\hline $\begin{array}{l}\text { T 2 Silvopastoral } \\
\text { Tradicional }\end{array}$ & Material Muerto & 44,9 & 14,2 & 8,1 & 17,4 \\
\hline T 3 Silvopastoral en Fajas & Material Muerto & 31,5 & 10,3 & 9,6 & 13,1 \\
\hline T 4 Ganadero Tradicional & Material Muerto & 40,5 & 21,1 & 5,7 & 14,0 \\
\hline
\end{tabular}

Como se observa en el Cuadro $\mathrm{N}^{\circ} 17$ y en la Figura $\mathrm{N}^{\circ} 7$, este material se redujo fuertemente a partir de la segunda temporada, producto de un adecuado manejo de la pradera y animales y las fertilizaciones aplicadas.

En el Cuadro $\mathrm{N}^{\circ} 18$ se observa que solo el efecto temporada es significativo y los efectos tratamiento y tratamiento*tiempo no son significativos para la variable composición de material muerto. 


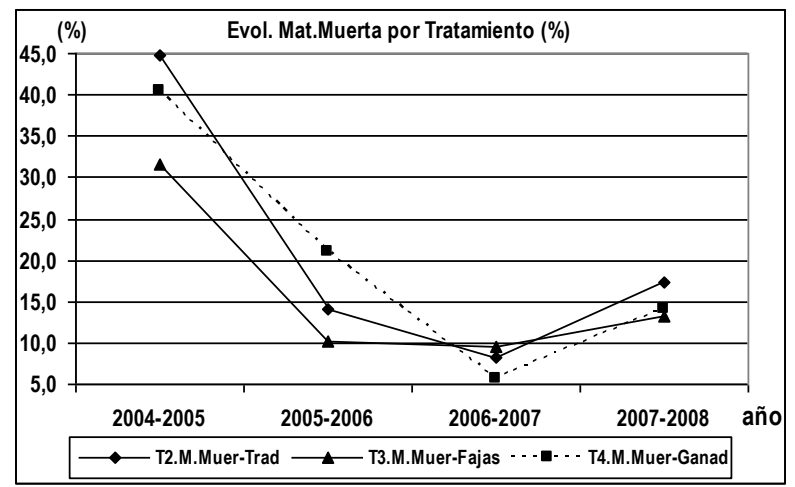

Figura $\mathrm{N}^{\circ} 7$

EVOLUCIÓN COMPOSICIÓN DE MATERIAL MUERTO POR TRATAMIENTO Y AÑO

Cuadro $\mathrm{N}^{\circ} 18$

RESULTADO ANÁLISIS DE VARIANZA PARA COMPOSICIÓN DE MATERIAL MUERTO (\%)

\begin{tabular}{|c|c|c|c|c|c|}
\hline F.V. & SC & gl & CM & $\mathbf{F}$ & p-valor \\
\hline Tratamiento & 400,63 & 2 & 200,31 & 2,97 & 0,0581 \\
\hline Temporada & 10222,02 & 3 & 3407,34 & 50,55 & $<0,0001$ \\
\hline Tratamiento*Temporada & 709,76 & 6 & 118,29 & 1,76 & 0,1222 \\
\hline Error & 4448,46 & 66 & 67,40 & & \\
\hline Total & 15806,46 & 77 & & & \\
\hline
\end{tabular}

De acuerdo a resultados expuestos en el Cuadro $N^{\circ} 19$ y en la Figura $N^{\circ} 7$, se observa que no existen diferencias significativas en el tiempo entre los tratamientos, con valores de materia muerta levemente superiores para los sistemas silvopastorales, por una mayor proporción de acículas de pino.

\section{Cuadro $\mathrm{N}^{\circ} 19$}

RESULTADO ANÁLISIS DE VARIANZA PARA EFECTO TRATAMIENTO EN MATERIAL MUERTO

\begin{tabular}{|l|r|r|c|}
\hline Tratamiento & Medias & $\mathbf{n}$ & $\begin{array}{c}\text { Significancia } \\
\mathbf{p}<=\mathbf{0 , 0 5 ^ { * }}\end{array}$ \\
\hline T 2 Silvopastoral Tradicional & 21,29 & 26 & $\mathrm{~A}$ \\
\hline T 4 Ganadero Tradicional & 20,33 & 25 & $\mathrm{~A}$ \\
\hline T 3 Silvopastoral en Fajas & 16,11 & 27 & $\mathrm{~A}$ \\
\hline
\end{tabular}

*Letras distintas indican diferencias significativas $(p<=0,05)$

Para el efecto tiempo (Cuadro $\mathrm{N}^{\circ} 20$ ), la temporada inicial presenta mayores valores de material muerto, con diferencias significativas respecto a las otras temporadas. Lo anterior debido a que en ese periodo había mayor presencia de este material por un mal manejo anterior, en el caso ganadero, y por la presencia anterior de una alta densidad de árboles en los tratamientos silvopastorales, lo cual originaba un mayor depósito de hojarasca forestal, disminuyendo en temporadas posteriores debido al manejo forestal realizado.

En la temporada 2006-2007 se presentaron los menores valores de material muerto, con diferencias estadísticas en relación a las otras temporadas. 


\section{Cuadro $\mathrm{N}^{\circ} 20$}

RESULTADO ANÁLISIS DE VARIANZA PARA EFECTO TIEMPO EN MATERIAL MUERTO

\begin{tabular}{|c|r|c|c|}
\hline \multicolumn{1}{|l|}{ Temporada } & Medias & $\mathbf{n}$ & Significancia $\mathbf{p}<=\mathbf{0 , 0 5}{ }^{\boldsymbol{*}}$ \\
\hline $2004-2005$ & 38,97 & 18 & $\mathrm{~A}$ \\
\hline $2005-2006$ & 15,18 & 20 & $\mathrm{~B}$ \\
\hline $2007-2008$ & 14,84 & 20 & $\mathrm{~B}$ \\
\hline $2006-2007$ & 7,99 & 20 & $\mathrm{C}$ \\
\hline
\end{tabular}

*Letras distintas indican diferencias significativas $(p<=0,05)$

\section{Distribución de la Producción en Materia Seca}

En el siguiente análisis se presentan los resultados de las evaluaciones de los transectos, realizados para evaluar la composición de la pradera bajo el efecto de los árboles en los tratamientos silvopastorales. En su evaluación y análisis de realiza un análisis descriptivo.

\section{-Sistema Silvopastoral Tradicional}

Como se observa en la Figura $N^{\circ}$ 8, se genera una curva normal en la distribución de la producción de la pradera, bajo la influencia arbórea en el sistema tradicional.

La producción disminuye un 30\% en situación bajo copa de los árboles (BCO y BCE), y un 7\% a semisombra (SSO y SSE), con respecto a una situación relativamente abierta (AA-2,5 m, Figura $N^{\circ} 9$ ) sin la influencia directa del árbol (promedio de $A O$ y $A E$ ). No se presentan diferencias con respecto a la ubicación este $(\mathrm{E}) \mathrm{u}$ oeste $(\mathrm{O})$.

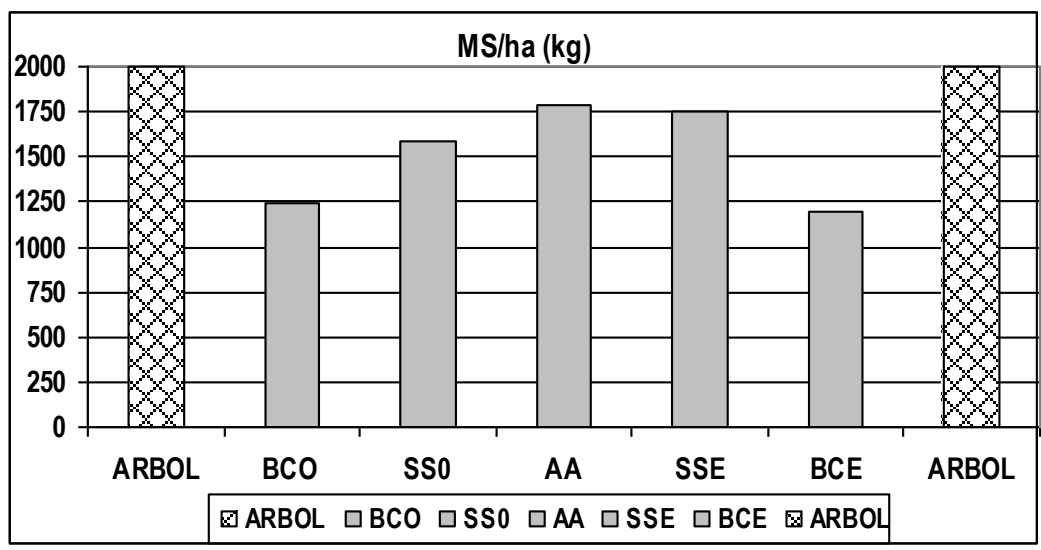

\section{Figura $\mathbf{N}^{\circ} 8$}

DISTRIBUCIÓN DE LA PRADERA EN TRATAMIENTO SILVOPASTORAL TRADICIONAL POR EFECTO DE LA CERCANÍA DE LOS ÁRBOLES

\section{-Sistema Silvopastoral en Fajas}

Para el tratamiento en fajas (Figura $\mathrm{N}^{\circ}$ ), se observa una distribución similar al sistema tradicional, con una producción menor mientras más cerca se encuentre de la copa de los árboles. 
La producción disminuye en un $29 \%$ para parcelas ubicadas a $1 \mathrm{~m}$ de la copa de árboles de la faja (O1 y E1) y en un 17\% para parcelas ubicadas a $4 \mathrm{~m}$ (O4 y E4), con respecto a la producción encontrada a 9 metros de la faja (O9 y E9).

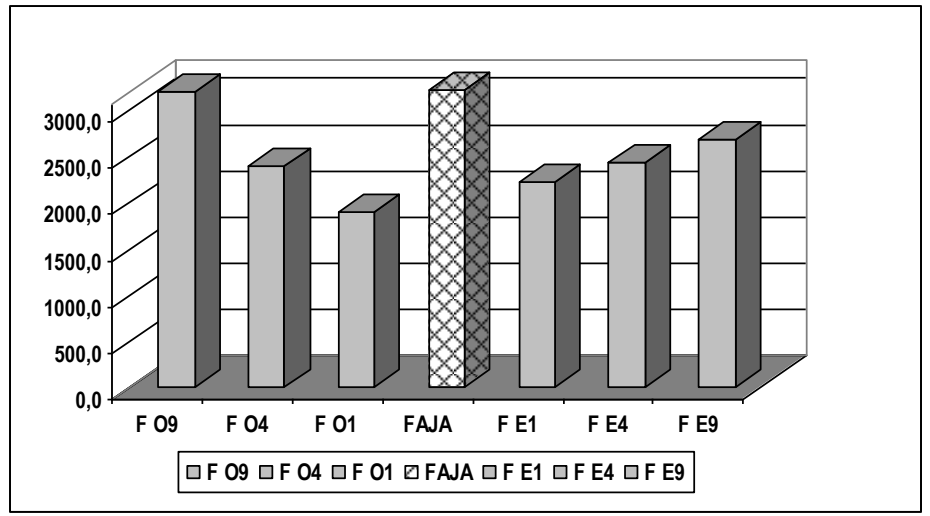

Figura $N^{\circ} 9$

DISTRIBUCIÓN DE LA PRADERA EN TRATAMIENTO SILVOPASTORAL EN FAJAS POR EFECTO DE LA CERCANÍA DE LA FAJA DE ÁRBOLES

\section{Distribución de la Composición de los Pastos}

\section{-Sistema Silvopastoral Tradicional}

La composición de la pradera en el sistema tradicional varía de acuerdo a la cercanía de las copas de los árboles, que en este caso se encuentran a un distanciamiento promedio de $5,2 \mathrm{~m}$ uno del otro. Para trébol blanco (leguminosa), a medida que se aleja de la copa aumenta su presencia, y para pasto ovillo (gramínea) disminuye (Figura $\mathrm{N}^{\circ} 10$ ). El material muerto, compuesto principalmente por acículas y ramillas pequeñas de los árboles, aumenta con la cercanía de las copas, y las malezas no son afectadas.

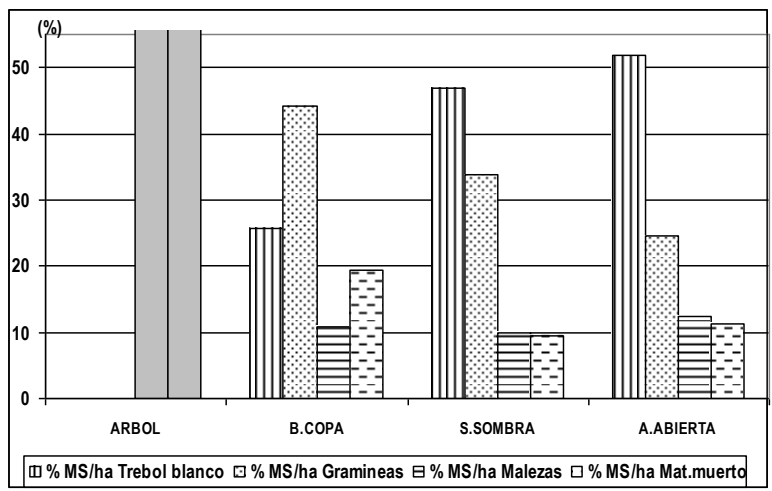

Figura $N^{\circ} 10$

DISTRIBUCIÓN DE LA COMPOSICIÓN BOTÁNICA DE LA PRADERA, EN RELACIÓN A LA CERCANÍA DE LOS ÁRBOLES EN EL SISTEMA SILVOPASTORAL TRADICIONAL (2007-008) 


\section{-Sistema Silvopastoral en Fajas}

En el caso de tratamiento en fajas, donde estas fajas se encuentran a una distancia una de otra de 21 a $22 \mathrm{~m}$ promedio, existe una tendencia en leguminosas (trébol blanco) a aumentar a una distancia de $4 \mathrm{~m}$ de la faja (O4 y E4), y disminuir cuando esta distancia aumenta a $9 \mathrm{~m}$ (O9 y E9). Las gramíneas aumentan con la cercanía a la faja (O1 y E1), y las malezas tienen una distribución sin una tendencia clara. El caso de material muerto, aumentó a medida que se aleja de la faja, lo cual es diferente al caso tradicional.

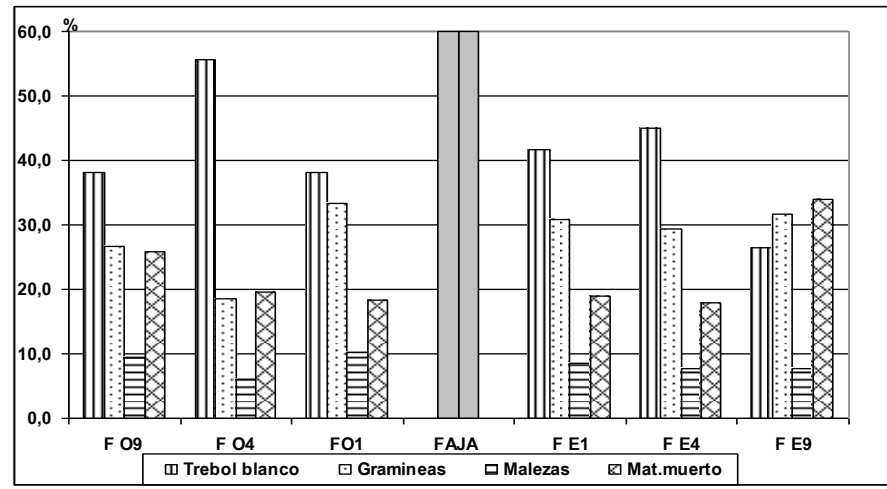

Figura $N^{\circ} 11$

DISTRIBUCIÓN DE LA COMPOSICIÓN BOTÁNICA DE LA PRADERA EN RELACIÓN A CERCANÍA DE FAJAS DE ÁRBOLES, EN EL SISTEMA SILVOPASTORAL EN FAJAS (2007-2008)

Para leguminosas, de acuerdo a estos resultados (tradicional y fajas), existe una tendencia a aumentar a una distancia de 4-5 m de los árboles y en gramíneas lo mismo a 1-2 m. No se encontraron diferencias entre ubicación este u oeste.

\section{DISCUSIÓN}

\section{Producción de la Pradera en Materia Seca}

De acuerdo a los resultados obtenidos, en las cuatro temporadas de evaluación, se ha visto un buen desarrollo del componente herbáceo en los dos sistemas con densidades silvopastorales, lo cual ha sido superior al sistema ganadero. La producción de la pradera en el tratamiento en fajas ha sido mayor a los otros dos tratamientos, con diferencias significativas.

En el sistema en fajas, los árboles dispuestos como cortinas cortavientos (Figura $\mathrm{N}^{\circ} 12$ ), otorgan una buena protección a la pradera, sin afectar, hasta la fecha de evaluación, su productividad.

Estos resultados indican que los sistemas silvopastorales se presentan como una buena alternativa para mejorar la productividad pratense, ofreciendo además efectos ambientales positivos en términos de protección de suelos y abrigo al ganado. 


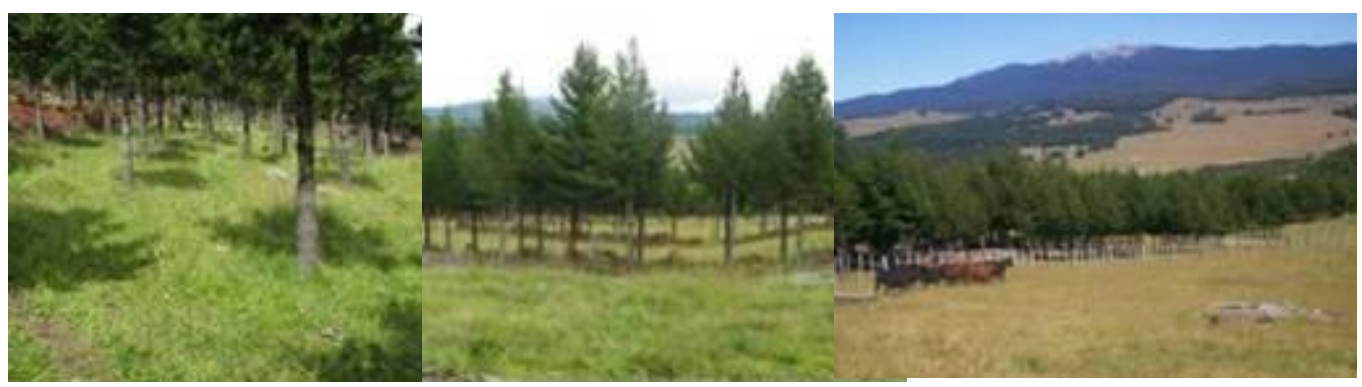

Figura $\mathrm{N}^{\circ} 12$

DESARROLLO DE LA PRADERA EN RELACIÓN A PRESENCIA DE ÁRBOLES, POR TRATAMIENTO: T2, DESARROLLO DE ÉSTA BAJO LA INFLUENCIA DIRECTA DE LOS ÁRBOLES (izq; T3, INFLUENCIA DE FAJAS SOBRE LA PRADERA SIMULANDO CORTINAS (centro); T4, PRADERA SIN LA INFLUENCIA DE ÁRBOLES (der).

Los resultados presentan una correlación positiva entre la productividad herbácea y las densidades arbóreas en los sistemas silvopastorales, lo cual no concuerda con los resultados obtenidos por otros autores (Lewis et al., 1983; Watson et al., 1984; Percival et al., 1984a; Peri et al., 2007), que indican que a medida que la densidad o cobertura de copa aumenta, disminuye la producción herbácea. Es posible que en el caso de este estudio, aún no se refleje esta tendencia de efecto de la competencia arbórea sobre la productividad de la pradera dado el corto periodo de evaluación, solo cuatro temporadas. Al final del periodo de evaluación, la cobertura de copa máxima alcanzada ha sido del $32 \%$ para silvopastoral tradicional y de $31 \%$ para fajas. Sin embargo, en las últimas temporadas se ha observado una tendencia a la disminución en su productividad, lo cual se debe seguir evaluando en próximas temporadas. Diversos autores indican que se produce una reducción importante de la producción herbácea cuando la cobertura de copa aumenta sobre el 40-50\% (Lewis et al., 1983; Wolters, 1974; Belesky et al., 2006; Peri et al., 2007).

Los resultados positivos de producción pratense en los tratamientos silvopastorales entregados en el presente estudio pueden deberse a los beneficios que otorga el componente forestal al modificar positivamente los factores climáticos en esta zona patagónica de Chile. Diversos autores mencionan la disminución del viento, el aumento de la temperatura del suelo y ambiental, y el aumento de la humedad relativa entre otros factores beneficiosos para el desarrollo de los vegetales, lo cual coincide con lo expuesto por Anderson et al. (1988), Sotomayor (1990b) y Polla (1998).

También, es probable que existiera al inicio del ensayo una buena cama de semillas de leguminosas y gramíneas bajo los árboles. Esto les habría permitido prosperar rápidamente luego de la apertura del dosel por la llegada de más luminosidad al suelo, sin mayor competencia y con el abrigo de los árboles que les proporcionaron las condiciones apropiadas para ello. Además, se debe considerar que en temporadas anteriores previas al inicio del ensayo, el manejo ganadero y pratense que el propietario le daba al sector ganadero, al parecer no fue el más apropiado, por lo que la recuperación de la pradera en ese caso ha sido más lenta.

Respecto de la reducción de la productividad herbácea en la última temporada, esta se debe principalmente a tres factores. El principal es la sequía que ocurrió en el sur de Chile durante la primavera y verano 2007-2008. En un año normal de precipitación para la zona de Coyhaique, en primavera-verano (entre septiembre-enero) la pluviometría es de $282,6 \mathrm{~mm}$ año-1 (Cuadro $\mathrm{N}^{\circ}$ 21), y esa temporada llovió solo $229,3 \mathrm{~mm}$ en la zona de Coyhaique (estación oficial Dirección General de Aguas DGA más cercana), con una déficit del $19 \%$. Si se compara estos valores oficiales con los registros de precipitación obtenidos en las estaciones climáticas ubicadas en los tratamientos, esta reducción fue del $34,5 \%$. 
Cuadro $\mathrm{N}^{\circ} 21$

PRECIPITACIÓN MENSUAL ESTACIÓN COYHAIQUE

\begin{tabular}{|c|c|c|c|c|c|c|c|c|}
\hline \multirow{2}{*}{ AÑO } & Sep & Oct & Nov & Dic & Ene & Feb & Mar & Abr \\
\hline & \multicolumn{8}{|c|}{$(\mathbf{m m})$} \\
\hline 20042005 & 77,1 & 83,4 & 40,4 & 77,9 & 44,6 & 13,1 & 114,4 & 123,8 \\
\hline $2005-2006$ & 11,9 & 76,5 & 103,1 & 3,6 & 87,7 & 23,9 & 98,6 & 113,7 \\
\hline 2006-2007 & 47,1 & 100,1 & 45,7 & 111,9 & s.i & s.i & s.i & s.i \\
\hline $2007-2008$ & 48,7 & 82,9 & 17,3 & 30,3 & 50,1 & s.i & s.i & s.i \\
\hline Media Mes* & 59,0 & 70,4 & 48,5 & 56,0 & 48,7 & 34,1 & 69,4 & 90,6 \\
\hline $\begin{array}{l}\text { Estación climática } \\
\text { tratamiento ganadero } \\
\text { temporada } 2007- \\
2008^{* *}\end{array}$ & s.i. & 53,6 & s.i. & 25,6 & 36,7 & 16,5 & 32,9 & 76,3 \\
\hline
\end{tabular}

* Estación Coyhaique (Escuela Agrícola) datos 1980 a 2008. Altitud 343 msnm; 45 34' 26 LS y 72 01' 43 LO

**Estación ubicada en tratamiento ganadero, Predio San Gabriel.

El segundo factor que puede haber afectado la productividad herbácea es la influencia del componente forestal en el desarrollo de la pradera en los sistemas silvopastorales. Es especialmente importante considerar la cobertura de copa, que aumentó de $15 \%$ a $32 \%$ en el sistema tradicional y de 24 a $31 \%$ en el tratamiento en fajas en las últimas temporadas, dado que este aumento de cobertura de copa afecta la cantidad de luz que recibe la pradera (Lewis et al., 1983; Watson et al., 1984; Percival et al., 1984b; Peri et al., 2007) y modifica otros factores climáticos como la velocidad del viento (Teuber et al., 2009) y temperatura (Percival et al., 1984b).

El tercer factor es la disminución de azufre en la última temporada de evaluación, apreciada en un análisis de fertilidad de los suelos, este elemento es deficitario en los suelos de la Región de Aysén y limita su productividad.

Se deberá analizar estas tendencias en temporadas futuras para corroborar lo expuesto en otros estudios, o correlacionar estos resultados con el efecto positivo de los árboles en ambientes fríos y ventosos, como es ésta zona austral de Chile.

\section{Composición de la Pradera}

En relación a la composición de pastos en la pradera, también se observa un efecto beneficioso de los árboles sobre las leguminosas y gramíneas. De acuerdo a los resultados expuestos (Figura $\mathrm{N}^{\circ} 4$ ) existe una mayor presencia de leguminosas en el tratamiento en fajas y tradicional, aunque no existen diferencias significativas con el ganadero. Sí se presentaron diferencias significativas, a favor de los tratamientos silvopastorales, para gramíneas (Cuadro $\mathrm{N}^{\circ}$ 11) con respecto al ganadero.

Esto indica, que la protección de los árboles sobre la pradera benefició el desarrollo de las leguminosas y gramíneas presentes en las temporadas evaluadas. Lo anterior puede estar relacionado con una mayor cobertura de copa, lo cual ha beneficiado con su protección el desarrollo de las leguminosas y las gramíneas.

Algunos autores han indicado que las leguminosas se adaptan mejor a la sombra para su desarrollo que las gramíneas. Lo anterior se ajusta a los resultados expuestos por Watson et al. (1984), encontrando en su estudio que las especies más tolerantes al efecto sombra fueron las leguminosas, como trébol subterráneo y trébol encarnado, con resultados superiores a las gramíneas, como ballica anual y festuca. Por otro lado, Percival et al. (1984a) encontraron que la cobertura de trébol blanco y ballica anual aumentaban con el tiempo, en cada año de medición 
entre los 2 y 9 años, creciendo bajo densidades de 100 a 400 arb ha $^{-1}$ de Pinus radiata, pero eran inferiores a una pastura abierta, y disminuían con un aumento de la densidad.

En estudio realizado por Lin et al. (1999), donde probaron el efecto de tres niveles de sombra ( $0 ; 50$ y $80 \%)$, sobre diversas especies de pastos y leguminosas, encontraron que pasto ovillo no tuvo diferencias de producción total entre 0 y $50 \%$ de sombra, y que esta disminuyó a menos de la mitad con $80 \%$ de sombra. Similar resultado encontraron con trébol blanco, sin diferencias entre 0 y $50 \%$ de sombra. Los resultados de Lin et al. (1999), concuerdan con lo encontrado en el presente estudio, con un aumento de leguminosas en los tratamientos silvopastorales, con cobertura entre $31-32 \%$, aunque no significativos, y con un aumento significativo en gramíneas, en relación al tratamiento ganadero con $0 \%$ de cobertura.

En cuanto a presencia de malezas y material muerto en los tres tratamientos estudiados en el presente trabajo, al parecer es un tema de manejo ganadero, dado que ambas redujeron su presencia luego de un adecuado manejo de los sistemas en el tiempo. Estos resultados son similares a lo expuesto por Percival et al. (1984a). En el futuro, se espera que la presencia de material muerto por acículas aumente con el aumento del desarrollo de las copas de los árboles en los tratamientos silvopastorales, lo cual debe ser manejado con podas y raleos y un adecuado manejo de desechos.

En relación a la distribución y composición de los pastos dentro de los tratamientos silvopastorales bajo la influencia de los árboles, se observó que las leguminosas prosperaron mejor al abrigo de los árboles, pero que aumentaron su presencia a medida que se alejaban del árbol, entre 2,5 a 5,0 m, con una menor proporción bajo la copa o a un metro de estas. En relación a las gramíneas, éstas aumentaron su presencia en las cercanías de los árboles, entre 1,0 a 2,5 m. Lo anterior es importante para la planificación del diseño de la densidad y distribución de los árboles en el sistema silvopastoral.

\section{CONCLUSIONES}

Sobre la base a los resultados encontrados en el presente estudio, se puede concluir que la introducción de árboles, bajo un sistema de manejo y ordenamiento silvopastoral, en terrenos ganaderos de la Región de Aysén de Chile, ha sido beneficiosa para la producción pratense en las temporadas evaluadas.

Los dos sistemas silvopastorales presentaron una mejor producción pratense, en relación al sistema ganadero sin árboles, especialmente el sistema silvopastoral en fajas.

En relación a la composición de pastos en la pradera, se verificó una mayor presencia de leguminosas en los tratamientos silvopastorales en fajas y tradicional, aunque no existieron diferencias significativas con el ganadero. Para gramíneas, se presentaron diferencias significativas, a favor de los tratamientos silvopastorales, con respecto al ganadero.

Las leguminosas se desarrollaron mejor al abrigo de los árboles, a una distancia de 2,5 a $5,0 \mathrm{~m}$, y con una menor proporción entre bajo copa y un metro de estas. En relación a las gramíneas, éstas aumentaron su presencia en las cercanías de los árboles, entre 1,0 a 2,5 m.

\section{REFERENCIAS}

Anderson, G., Moore, R. and Jenkins, P., 1988. The integration of pasture, livestock and widely-spaced Pine in South Western Australia. Agrofor. Syst. 6:195-211.

Belesky, D. P., Chatterton, N. J. and Neel, J. P. S., 2006. Dactylis glomerata growing along a light gradient in the central Appalachian region of the eastern USA: III. Nonstructural carbohydrates and nutritive value. Agrofor. Syst. (2006) 67:51-61. 
Benavides, R., Douglas, G., and Osoro, K., 2009. Silvopastoralism in New Zealand: review of effects of evergreen and deciduous trees on pasture dynamics. Agrofor. Syst. DOI 10.1007/S10457-008-9186-6.

Benedetti, S. y Valdés, J., 1996. Prácticas agroforestales tradicionales en la zona árida y semiárida de Chile. La Serena, Chile, Ministerio de Agricultura; Corporación Nacional Forestal. 65p.

DGA, 2008. Antecedentes estadísticos de pluviometría, Dirección General de Aguas, Región de Aysén, no publicados. Coyhaique, Chile.

Garret, H. E. and Buck, L., 1997. Agroforestry practice and policy in the United States of America. Forest Ecol. Manag. 91:5-15.

Garret, H. E., Kerley, M. S., Ladyman, K. P., Walter, W. D., Godsey, L. D., Van Sambeek, J. W. and Brauer, D. K., 2004. Hardwood silvopasture management in North America. Agrofor.Syst. 61:21-33.

INE, 2007. Censo Nacional Agropecuario. Instituto Nacional de Estadísticas. Chile

INFOR, 2008. Boletín Estadístico 121: Anuario Forestal 2008. Instituto Forestal, Santiago, Chile.

IREN-CORFO, 1979. Perspectivas de desarrollo de los recursos de la Región de Aysen del General Carlos lbañez del Campo: Suelos y Erosión, Caracterización Climática. Coyhaique, Chile. 113 pag.

Lewis, C., Burton, C., Monson, W. and Mccormick, W., 1983. Integration of Pines, pastures and cattle in southern Georgia, USA. Agrofor. Syst. 1:277-297.

Lin, C. H., Mc Graw, R. L., George, M. F. and Garret, H. E., 1999. Shade effects on forage crops with potential in temperate agroforestry practices. Agrofor.Syst. 44: 109-119.

Mead, D., 2009. Biophysical interactions in silvopastoral systems: A New Zealand perspective. En: Actas del $1^{\mathrm{er}}$ Congreso Nacional de Sistemas Silvopastoriles. Posadas, Misiones Argentina. Ed. Instituto Nacional de Tecnología Agropecuaria, Buenos Aires, Argentina. pp. 3-9.

Monfore, J. D., 1983. Livestock, a useful tool for vegetation control on Ponderosa Pine and Lodgepole Pine plantations. Pp.105-107. In: Forestland Grazing. Ed: by Roche, Jr. B.F. and Baumgrtner D. M., and Cooperative Extension, Wash. State Univ. Spokane, Washington.

Murgeitio, E., 2009. Aspectos relacionados con la sustentabilidad social y ambiental de los sistemas silvopastoriles en América tropical. En: Actas del $1^{\mathrm{er}}$ Congreso Nacional de Sistemas Silvopastoriles. Posadas, Misiones Argentina. Ed. Instituto Nacional de Tecnología Agropecuaria, Buenos Aires, Argentina. Pp. 66-69.

Nair, P. K. R., 1987. Soil productivity under agroforestry. In: Agroforestry Realities, Possibilities and Potentials. Ed. by Gholz H. L., Martinus Nijhoff and Junk W. Dordretch, The Netherlands.

Percival, N., Bond, D., Hawke, M., Andrew, B. and Knowles, R., 1984a. Effects of Radiate Pine pasture yields, botanical composition, weed production and production of a range of grass. In: Bibrough, G. W. (ed), Proceedings of a technical workshop of agroforestry. Ministry of Agriculture and Fisheries, Wellington. pp: 13-22

Percival, N., Hawke, M. and Andrew, B., 1984b. Preliminary report on climate measurements under Pinus radiata plants in farmland. In: Bibrough, G. W. (ed), Proceedings of a technical workshop of agroforestry. Ministry of Agriculture and Fisheries, Wellington. pp: 57-60

Peri, P., Lucas, R. and Moot, D., 2007. Dry matter production, morphology and nutritive value of Dactilys glomerata growing under different light regimes. Agrofor. Syst. 70: 63-79.

Polla, C., 1998. Estrategias de Acción en el Tema Silvopastoreo. En: Actas Seminario "Manejo Silvopastoral”, Trabajo N8, Young. Uruguay.

Snaydon, R. W. and Harris, P. M., 1979. Interactions belowground: The use of nutrients and water. In: Proc. International Workshop on Intercropping. Ed. by Willey, R. W. ICRISAT, Hyderabad, India. pp. 188-201.

Solangaarachchi, S. M. y Harper, J. L., 1987. The effect of canopy filtered light on the growth of White Clover, Trifolliun repens. Oecologia 72: 372-376. 
Sotomayor, A., 1989. Sistemas silvopastorales y su manejo. Documento Técnico № 42. Revista Chile Forestal, Diciembre 1989. CONAF. 8p.

Sotomayor, A., 1990a. Sistemas silvopastorales y su manejo. Chile Agrícola 157:203-206.

Sotomayor, A., 1990b. Bosques y forrajeras pueden complementarse (III Parte). Chile Agrícola 159:273-277.

Sotomayor, A. y Cabrera, C., 2008. Análisis de un sistema silvopastoral con Pinus radiata D. Don, asociado con ganado ovino en la zona mediterránea costera central de Chile. Ciencia e Investigación Forestal, Volumen 14 № 2 . pp: $269-286$.

Sotomayor, A., 2009. Sistemas silvopastorales, alternativa productiva para un desarrollo sustentable de la agricultura en Chile. En: Actas del $1^{\text {er }}$ Congreso Nacional de Sistemas Silvopastoriles. Posadas, Misiones Argentina. Ed. Instituto Nacional de Tecnología Agropecuaria, Buenos Aires, Argentina. pp.26-47.

Sotomayor, A., Teuber, O. y Moya, I., 2009. Resultados y experiencia sobre manejo de sistemas silvopastorales en la Región de Aysén. En: Sistemas Agroforestales para la Región de Aysén: Cortinas Cortaviento y Silvopastoreo. pp: 165-199. Ed: Teuber, O. Instituto de Investigaciones Agropecuarias e Instituto Forestal. Coyhaique, Chile.

Teuber, O., Moya, I., y Sotomayor, A., 2009. Resultados y experiencia en producción de cultivos forrajeros con cortinas adultas en la Región de Aysén. En: Sistemas Agroforestales para la región de Aysén: Cortinas Cortaviento y Silvopastoreo. pp: 85-128. Ed: Teuber, O. Instituto de Investigaciones Agropecuarias e Instituto Forestal. Coyhaique, Chile.

Teuber, O. y Ganderatz, S., 2009. Características geográficas y edafoclimáticas de la Región de Aysén. En: Sistemas Agroforestales para la Región de Aysén: Cortinas Cortaviento y Silvopastoreo. pp: 85-128. Ed: Teuber, O. Instituto de Investigaciones Agropecuarias e Instituto Forestal. Coyhaique, Chile.

Torres, F., 1983. Role of woody perennials in animal agroforestry. Agrofor. Syst. 1:131-163.

Watson, V. H., Pearson, H. A., Knight, W. E. and Hagedorn, C., 1984. Cool season forages for use in Pine forests. Pp.79-88. In: Agroforestry in the Southern Unites States, $33^{\text {rd }}$ Annual Forestry Symposium. Ed. by Linnartz, N. E. and Johnson, M. K. Louisiana Agric. Exp. Sta., Baton Rouge, Louisiana.

Wolters, G. L., 1974. Longleaf Uniola and Spike Uniola require shade. J. Range Manage. 34: 494-497. 